\title{
A new tool to target DNA repair
}

DNA repair systems are defective in many types of cancers, but deficiency in one repair pathway generally results in dependence on a compensatory repair pathway. Double-strand

These two papers suggest that alt-NHEJ and HR are competing pathways and compensate for each other so that HR-deficient cells activate alt-NHEJ and vice versa break (DSB) repair mostly occurs through two pathways: homologous recombination (HR) or nonhomologous end-joining (NHEJ). Two studies have shown that DNA polymerase- $\theta$ (POLQ) promotes an alternative form of NHEJ (alt-NHEJ) in mammalian cells and that its activity is essential for the survival of cells deficient in HR.

Telomeres can fuse in the absence of protective mechanisms. Mateos-Gomez et al. were interested in studying whether the sequence at the junction between two fused telomeres differed depending on the type of repair pathway used.

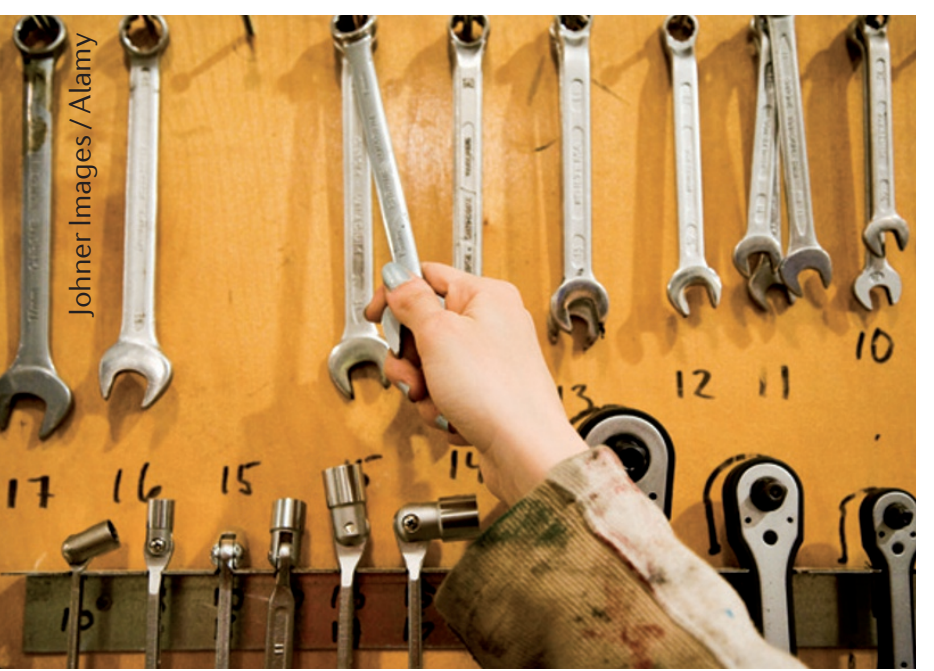

They found that random nucleotide insertions occurred at the junction of telomeres fused by alt-NHEJ. They then observed that these insertions were carried out by POLQ and that the activity of POLQ is specific to alt-NHEJ. Accumulation of POLQ at laser-induced DSBs was reduced after depletion of poly(ADP-ribose) polymerase 1 (PARP1) with small interfering RNAs (siRNAs) or with a PARP1 inhibitor, which suggests that PARP1, previously known to be required for alt-NHEJ, recruits POLQ to DSBs.

Next, the authors tested whether alt-NHEJ had a role in the survival of cells with impaired HR. They showed that depleting POLQ expression in HR-deficient mouse cells (harbouring mutations in either Brcal or Brca2) increased chromosomal aberrations and reduced cell survival. This suggests that alt-NHEJ mediated by POLQ promotes the survival of cells with impaired HR.

Ceccaldi et al. examined changes in DNA polymerase activity between tumour and normal tissue and observed that POLQ is overexpressed in high-grade serous ovarian cancer, which is often associated with $\mathrm{HR}$ deficiency and mutations in BRCA1 and BRCA2. Knockdown of POLQ in U2OS human osteosarcoma cells increased HR efficiency, as shown by the accumulation of RAD51 - a member of the HR pathway - at DNA breaks, suggesting that POLQ inhibits HR. Further experiments revealed that RAD51 binds to POLQ and that this binding is enhanced by exposure to ultraviolet radiation, suggesting that POLQ suppresses HR in cells under replicative stress. They also observed that POLQ-depleted cells have hypersensitivity to certain DNA-damaging agents, and that this hypersensitivity is higher when POLQ reduction is combined with HR deficiency caused by either depletion of Fanconi anaemia complementation group D2 (FANCD2, a protein also involved in $\mathrm{HR}$ ) or mutations in BRCA1. Indeed, Fancd $2^{-/-}$Polq $^{-/-}$mouse embryos showed severe congenital malformations, and Fancd2 $2^{--}$Polq ${ }^{-1-}$ mouse embryonic fibroblasts showed hypersensitivity to PARP inhibition.

These two papers suggest that alt-NHEJ and HR are competing pathways and compensate for each other so that HR-deficient cells activate alt-NHEJ and vice versa. As POLQ is a crucial factor in alt-NHEJ, POLQ inhibition might be a therapeutic option for cancers that harbour deficiencies in HR. Furthermore, as POLQ suppresses HR, tumours that overexpress POLQ are likely to be sensitive to PARP inhibitors.

M. Teresa Villanueva

ORIGINAL RESEARCH PAPERS Mateos-Gomez P. A. et al. Mammalian polymerase $\theta$ promotes alternative NHEJ and suppresses recombination. Nature 518, 254-257 (2015) |Ceccaldi, R. et al. Homologous-recombination-deficient tumours are dependent on Pol $\theta$-mediated repair. Nature 518, 258-262 (2015)

FURTHER READING Kent, T. et al. Mechanism of microhomology-mediated end-joining promoted by human DNA polymerase $\theta$. Nature Struct. Mol. Biol. http://dx.doi.org/10.1038/nsmb.2961 (2015) 